\title{
EXPERIENCIAS DE RECUPERACIÓN TERRITORIAL A Partir De Resistencias Al Modelo De PRODUCCIÓN FORESTAL
}

\section{Manuel Fuenzalida Díaz ${ }^{1}$ Simón Arce $^{2}$}

\section{Resumen}

Se describen diversas experiencias de recuperación territorial a partir de resistencias al modelo de producción forestal de monocultivo en comunidades mapuche lafkenche de Tirúa, en el litoral sur de la región del Biobío, Chile. Las resistencias adquieren mayor valor, sobre todo, porque en el periodo comprendido entre 1997 y 2014, la proporción de superficie comunal cubierta por plantación forestal aumentó de $33 \%$ a $45,4 \%$. A través del trabajo desarrollado en terreno durante los últimos cuatro años, ha sido posible rescatar historias de resistencias en distintas comunidades, como lo son la Comunidad Kellgko en la localidad de San Ramón, la Comunidad Lorenzo Quintrileo, en la localidad de Quidico, la Comunidad Milla Rayén en la localidad de Las Misiones y, finalmente, la Comunidad Alto Primer Agua.

\section{Introducción}

En este capítulo se describen diversas experiencias de recuperación territorial a partir de resistencias al modelo de producción forestal en comunidades mapuche lafkenche de Tirúa, en el litoral sur de la región del Biobío [ $38^{\circ} 20^{\prime} \mathrm{S}$ y $73^{\circ} 30^{\prime} \mathrm{W}$ ], Chile. Esta zona está dominada por un modelo forestal de monocultivo, heredado del Decreto Ley 701 de 1974, promulgado por la dictadura militar de Pinochet, que mantiene en constante tensión a las comunidades con el Estado y los empresarios (Donoso et al, 2015).

Este modelo industrial de plantación de árboles como pinos (pinus radiata) y eucaliptus (eucaliptus globulus) -especies exóticas de rápido

1 Departamento de Geografía, Universidad Alberto Hurtado, Chile. mfuenzal@uahurtado.cl

${ }^{2}$ Laboratorio Territorio y Sociedad. Universidad Alberto Hurtado contacto@labtys.cl

B ANUARI DEL CONFLICTE SOCIAL 2018 
crecimiento- en extensas áreas geográficas, tiene como finalidad abastecer mercados externos de madera, carbón, leña, troncos y celulosas (Gonzalez, 2015). Esto, supone el desplazamiento sistemático del bosque nativo y diversos efectos medioambientales en los territorios de las comunidades mapuche como: acidificación de los suelos, pérdida de nutrientes y agotamiento de los recursos hídricos, lo que se traduce en una creciente desertificación del territorio, pérdida de la riqueza natural, deterioro de la biodiversidad y destrucción de ecosistemas (Programa Chile Sustentable, 2004).

Actualmente, las políticas públicas, los programas y proyectos, en general, tienen una lógica de construcción e intervención social de relaciones asimétricas de poder entre Estado y comunidad (Martínez, 2006), en el que no se da una articulación efectiva entre las estructuras y funcionamientos de las instituciones y las comunidades indígenas (Martínez, 2006; Rubilar \& Roldan, 2014). Tal lógica es generalizada en los modelos de producción extractiva en América Latina, generando profundos conflictos socioambientales y niveles agudos de injusticia ambiental, en particular, en comunidades indígenas que habitan zonas rurales ricas en recursos naturales, profundizando el enraizamiento de relaciones asimétricas entre comunidad y Estado.

En particular, para la comuna de Tirúa, el 48\% de la superficie total comunal es de uso forestal (Censo Forestal Agropecuario, 2007), perteneciendo a dos grandes empresas del rubro: Mininco y Arauco. Ello ha significado conflictos territoriales producto de la ocupación de predios indígenas por parte de empresas forestales, lo que ha originado procesos de resistencia en contra de la expansión del monocultivo y defensa de la naturaleza, "enmarcada en la reconstitución y reemergencia de las identidades étnicas como defensa y preservación ante procesos de desestructuración materiales e inmateriales de las condiciones de reproducción sociocultural" (Pineda, 2012:182).

La explotación de territorios ancestrales ha dado origen a conflictos entre el desarrollo del sector forestal y la preservación del bosque nativo (PLADECO Tirúa, 2009) o mawida y, por lo tanto, de la reproducción social, material y cultural del Pueblo Mapuche, en relación a su cosmovisión y concepción de territorio. De acuerdo a la 
cosmovisión mapuche, todos los árboles y toda la naturaleza son sagrados y, desde esa visión, la explotación forestal representa la pérdida del espíritu del bosque y de la cultura ancestral. Por ello, existe un daño directo a la producción social y simbólica del pueblo, puesto que amenaza el carácter simbólico e identitario que las comunidades tienen con la tierra y con los elementos naturales como el mawida. De esta manera, la relación con la naturaleza busca el equilibrio a través de una relación armónica, donde el Pueblo Mapuche, antes de llevar a cabo cualquier trabajo que interfiera con ella -ya sea cortar un árbol o iniciar una siembra- pide permiso a ngenechen -O "cuidador de los hombres"-, a quien también deben agradecer (FUCOA, 2014). En efecto, todo territorio es sagrado y concebido "no solo como un espacio geográfico donde se habita, sino como ámbito en el que los seres humanos y la naturaleza constituyen un todo indivisible, un círculo equilibrado de vida. La relación seres humanos y naturaleza se piensa circular, armónica y basada en el principio de la reciprocidad se da y se recibe a la vez-" (Agosto \& Briones, 2007:296).

De esta manera, la relación con la naturaleza se encuentra regida por una serie de nociones simbólicas, junto a una serie de principios ecológicos y culturales que representan los pilares fundamentales de la noción de küme mongen o "buen (con)vivir" (Hasen, 2014). En base a esto último, es que la pérdida de suelo y de bienes naturales como el agua y el bosque nativo representan, para las comunidades mapuche, una situación medioambiental que influye en la salud de la comunidad (PLADECO Tirúa, 2009). En este sentido, es necesario entender que la cosmovisión del Pueblo Mapuche es el punto de partida desde el cual se debe entender la salud y el modelo medicinal ancestral, pues implica una relación directa entre el ser humano y su entorno, conformando una unión armónica indisoluble (Hasen, 2012).

Por ello es que diversos actores locales advierten la necesidad urgente de reforestar con especies nativas en espacios que presentan altos niveles de erosión, para recuperar suelos y proteger el agua para consumo humano. De esta manera, se plantea la preocupación por mantener un uso sustentable del bosque nativo para contribuir al equilibrio del territorio desde un enfoque ecosistémico e intercultural. Este enfoque tiene la "capacidad de articular de manera armónica el 
medioambiente, la sociedad humana y la economía, entendiendo los ecosistemas en sus vinculaciones al desarrollo humano" (Hasen, 2012:105).

Por todo lo anterior, este documento pretende describir diversas iniciativas de resistencia al monocultivo forestal en territorios mapuche lafkenche. Aquí, la apuesta es poner en valor la sabiduría de la comunidad por sobre el saber del "experto". En el apartado siguiente se detalla la evidencia de la expansión del modelo de producción forestal. Luego se describen historias de resistencias al modelo de producción forestal, para terminar con una sección de ideas al cierre.

\section{Evidencia de la expansión del modelo de producción forestal de monocultivo en Tirúa}

A partir de información oficial de la Corporación Nacional Forestal (CONAF), correspondiente al "Catastro y evaluación de los recursos vegetacionales de Chile", se procedió a cuantificar y espacializar las variaciones de la cobertura de uso de suelo en la comuna de Tirúa para los años 1997, 2008 y 2014. Es importante advertir aquí, que estas fechas son las únicas disponibles para el monitoreo, corrección cartográfica y actualización del catastro de los recursos vegetacionales. La relevancia de esta contribución estriba en que, por primera vez, se puede hacer un acercamiento (zoom) para la comuna de Tirúa.

A pesar de que, originalmente, son trece las categorías de uso de suelo posibles de espacializar, se consideró agrupar en seis nuevas categorías de análisis los usos de suelo presentes en Tirúa (ver Tabla 1). El software utilizado para la realización de los cálculos corresponde a ArcGis 10.1 y la extensión Patch Analyst 5.2, que facilita el análisis espacial de parches de paisajes o usos de suelo, y el modelado de atributos asociados con parches. Los resultados pueden ser observados en la Figura 1 y la Tabla 2.

\section{Tabla 1. Descripción de los criterios de clasificación para el área de estudio, en base a información del "Catastro de usos de suelo" de la CONAF.}

\section{B ANUARI DEL CONFLICTE SOCIAL 2018}

DOI. 10.1344/ACS2019.9.9 


\begin{tabular}{|l|l|}
\hline Categoría de análisis & Descripción \\
\hline Cuerpos de agua & $\begin{array}{l}\text { Trata a los "Cuerpos de agua" y "Humedales". } \\
\text { Esencialmente bajo el precepto de considerarse zonas } \\
\text { con presencia de recurso hídrico. }\end{array}$ \\
\hline Terrenos agrícolas & $\begin{array}{l}\text { Se mantiene su nomenclatura. Considera las categorías de } \\
\text { uso destinadas a la agricultura. }\end{array}$ \\
\hline Matorral/pradera & $\begin{array}{l}\text { Agrupa los usos de suelo correspondientes a: "Praderas", } \\
\text { "Matorral", "Matorral-pradera". Se caracterizan por } \\
\text { presencia de especies arbustivas, de alta densidad y baja } \\
\text { altura. }\end{array}$ \\
\hline Bosque Nativo & $\begin{array}{l}\text { Reúne las siguientes categorías: "Bosque mixto", } \\
\text { "Renoval", "Bosque Nativo Adulto", "Bosque Nativo } \\
\text { Adulto-Renoval". Estas corresponden al tipo de Bosque } \\
\text { Húmedo Valdiviano. }\end{array}$ \\
\hline Plantación Forestal & $\begin{array}{l}\text { Considera el criterio "Plantaciones". Corresponde a suelo } \\
\text { con plantación de especies arbóreas exóticas, } \\
\text { principalmente Pinus Radiata Eucalyptus. }\end{array}$ \\
\hline Otros usos & $\begin{array}{l}\text { Se incorporan "Áreas sin vegetación" y "Áreas Urbanas- } \\
\text { Industriales". Su justificación se da por su poca } \\
\text { asociatividad con otras categorías, además de una baja } \\
\text { presencia en el área de estudio. }\end{array}$ \\
\hline
\end{tabular}

Fuente: Elaboración propia.

En primera instancia, en la Figura 1es posible ver un reducido y fragmentado bosque nativo ubicado principalmente en torno a las quebradas y cursos hídricos, cuestión concordante con lo estipulado en la Ley 20.283. Destacan los casos del estero Medihueico en las cercanías de Puerto Choque, los bordes del estero Paillaco, las inmediaciones del río Tirúa, además de importantes parches en el sureste como en el sector de Loncotripai, el río Matranquil, estero Los Maquis y La Campana. En términos de cambios en su distribución, se aprecia un mantenimiento de su área, aunque con leves reducciones en su extensión.

El matorral/pradera tendría inicialmente una alta presencia espacial, ubicándose principalmente en dos flancos: hacia el sur del río Tirúa, y la otra, al norte, entre la laguna Butaco y el lago Lleu lleu. Sin embargo, entre los años 2008 y 2014 se ve un significativo reemplazo de esta clase en ambos sectores. Al norte, con predominio de los terrenos agrícolas y, al sur, con plantación forestal. 
Figura 1. Variación espacio-temporal de los usos de suelo en Tirúa
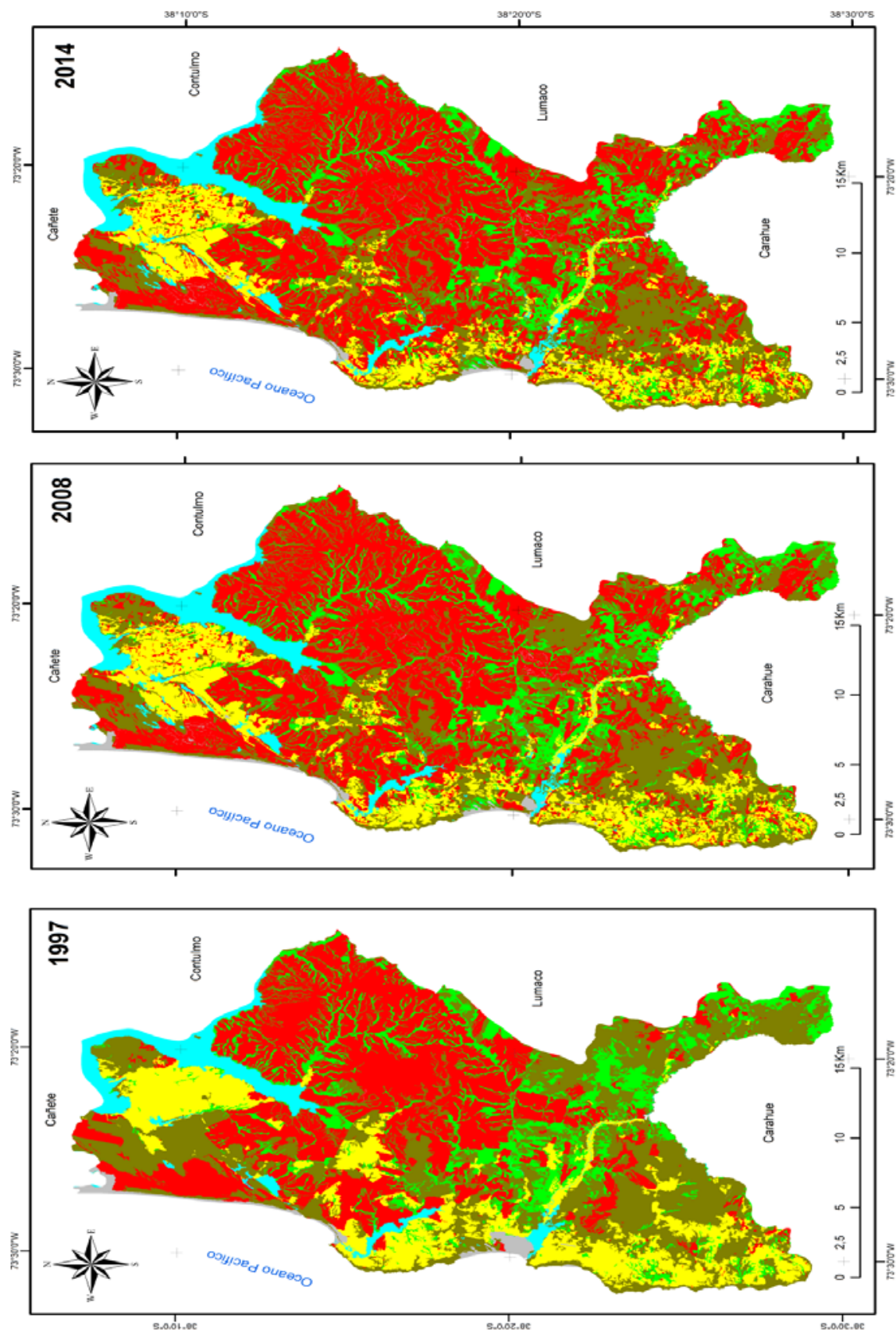

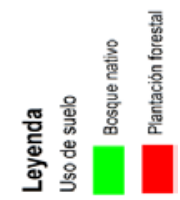

Fuente: Elaboración propia. 
Tabla 2. Área y proporción según clase de uso de suelo

\begin{tabular}{|l|r|r|r|}
\hline Class Area (CA) & \multicolumn{1}{|c|}{$\mathbf{1 9 9 7}$} & \multicolumn{1}{c|}{$\mathbf{2 0 0 8}$} & \multicolumn{1}{c|}{$\mathbf{2 0 1 4}$} \\
\hline Cuerpos de agua & 3.009 & 3.127 & 3.127 \\
\hline Terrenos agrícolas & 9.591 & 8.378 & 7.391 \\
\hline Matorral/pradera & 19.911 & 17.366 & 15.703 \\
\hline Bosque nativo & 8.470 & 7.531 & 7.304 \\
\hline Plantación forestal & 20.841 & 25.522 & 28.398 \\
\hline Otros usos & 775 & 674 & 675 \\
\hline Proporción (\%) & & & \\
\hline Cuerpos de agua & 4,8 & 5,0 & 5,0 \\
\hline Terrenos agrícolas & 15,3 & 13,4 & 11,8 \\
\hline Matorral/pradera & 31,8 & 27,7 & 25,1 \\
\hline Bosque nativo & 13,5 & 12,0 & 11,7 \\
\hline Plantación forestal & 33,3 & 40,8 & 45,4 \\
\hline Otros usos & 1,2 & 1,1 & 1,1 \\
\hline
\end{tabular}

Fuente: Elaboración propia.

Vale destacar que, sin lugar a dudas, el uso de suelo que mayores implicancias en cuanto a su extensión espacial, como también, por sus consecuencias morfológicas en el paisaje, son las plantaciones forestales. En cuanto a su distribución, se materializa inicialmente una concentración en el noreste de la comuna, mientras que para los años 2008 y 2014 se da una expansión unidireccional a expensas de la mayoría de los otros usos de suelo.

Por su parte, los terrenos agrícolas se ubicaban, para 1997, en su mayoría hacia el oeste del lago Lleu Lleu. También en los alrededores de Quidico: al sur en Ponotro y, al este, camino a Paillaco. Al sur de Tirúa también se concentraría el fenómeno: Bajo la Guerra, El Molino y Quilantahue mantenían dinámicas agrícolas. En términos generales, se mantuvieron en el sector costero del área de estudio, incorporándose, con el paso del tiempo, fragmentos de plantaciones forestales en su estructura. 
Los grandes cuerpos de agua estarían ubicados hacia el sector costero, como el río Tirúa y sus humedales adyacentes, las zonas inundables del Estero Chalamía, laguna Butaco y la laguna Quidico. Además, el lago Lleu Lleu, al norte, se posiciona como el mayor cuerpo de agua en el área. Ahora bien, en términos de cambio en este lapso temporal, parecieran no sufrir modificación espacial significativa.

Finalmente, la categoría denominada "Otros usos" apenas se visualiza espacialmente, pues representa a las pequeñas zonas urbanas correspondientes a los poblados de Tirúa y Quidico, además de una estrecha franja en el sector costero asociado a suelo desprovisto de vegetación.

\section{Historias de resistencias al modelo de producción forestal}

En la comuna de Tirúa existen diversas experiencias de recuperación territorial a partir de resistencias al modelo forestal, protagonizadas por hombres y mujeres mapuche que le dicen "basta" a la explotación de "palos" que destruyen su mawida y los privan de agua.

A través del trabajo desarrollado en terreno durante los últimos cuatro años, posibilitado por una estrategia de Aprendizaje Servicio entre la Carrera de Geografía de la Universidad Alberto Hurtado y la I. Municipalidad de Tirúa, y la gran colaboración de Susana Huenul Colicoy que, desde la Unidad de Familia del Departamento de Desarrollo Comunitario de la Municipalidad de Tirúa, ha impulsado acciones como la protección del agua, las semillas y la reforestación del bosque nativo, es posible rescatar historias de resistencias en distintas comunidades, como los son la Comunidad Kellgko en la localidad de San Ramón (Sector Norte); Comunidad Lorenzo Quintrileo, en la localidad de Quidico (Sector Centro); Comunidad Milla Rayen en la localidad de Las Misiones (Sector Sur); y, finalmente, Comunidad Alto Primer Agua (Sector Sur).

A continuación se presentan algunos de estas historias, las cuales permiten comprender y reconocer las necesidades y dinámicas territoriales, en un contexto local sobre el impacto del monocultivo forestal. 


\section{Historia de resistencia 1: Víctor Javier Mariñan Pilquiman, Comunidad Kellgko}

Víctor nació en 1974 en el sector de San Ramón en la comuna de Tirúa. Cursó su enseñanza básica en la escuela rural del sector, en donde también creció y conoció lo referente a la cultura mapuche de la cual es parte. Realizó su enseñanza media en la comuna de Angol, en donde se tituló de Técnico Mecánico Industrial. En estos años fue cuando comenzó a mostrar interés por ser dirigente político, puesto que fue elegido representante estudiantil de su colegio y luego, a nivel comunal. Al terminar la enseñanza media, emigra a Santiago en busca de mejores oportunidades laborales, realizando previamente su práctica laboral en la comuna de Cañete. En Santiago ingresa a la educación superior para estudiar automatización, estudios que no logra completar. El año 2006 decide volver a su comunidad de origen, en donde es activo participante de las reivindicaciones de su pueblo y su cultura. La decisión de volver a sus tierras tiene que ver con que Víctor toma conciencia de la situación histórica de su pueblo, por lo que se presenta para ser dirigente social de sus compañeros de comunidad y representarlos en las diversas instancias de diálogo y conflicto que se avizoran. Sin embargo, muchas de estas ilusiones de construir política desde la base comunitaria mapuche se vieron mermadas por un lamentable accidente a caballo el año 2007, que lo mantuvo cerca de un año postrado, sin poder trabajar ni representar a su pueblo. Sin embargo, esta situación le dio más fuerza y ánimo para encarar la lucha social. Cuando Víctor vuelve a la comunidad, esta se hallaba profundamente dividida y era reacia a participar en acciones reivindicativas en el territorio. Víctor logra conciliar estas diferencias y es elegido para el cargo de presidente de la comunidad Kellgko, el año 2010. Actualmente ejerce su segundo periodo de presidente de la comunidad, que finaliza este 2019. Entre los principales logros de la comunidad -con él como dirigente- se cuenta el proceso de recuperación del predio denominado Pilkitue, predio que al día de hoy es controlado y gestionado por la comunidad y sus integrantes.

Dentro de las situaciones negativas que le han traído el hecho de ser dirigente social, él recuerda el allanamiento sufrido en su hogar, en 2016, para buscar armas. Víctor considera que dichos allanamientos 
tienen por fin amedrentarlo para que desista de sus acciones que están encaminadas a combatir el monocultivo forestal y recuperar la identidad lafkenche. A continuación, se muestran dos citas extraídas de la entrevista realizada a Víctor:

"Si bien los allanamientos que tuve me afectaron personal y familiarmente, me han dado más fuerza para seguir en la lucha de mi pueblo" (Cita 1)

"Yo sueño que algún día podamos vivir del mar y navegar en él, con mi compadre Liempi y otros integrantes de la comunidad" (Cita 2)

\section{Historia de resistencia 2: Teresa Quintrileo, Comunidad Lorenzo Quintrileo}

Teresa ha vivido siempre en Tirúa-Quidico. Vive con su compañero, Segundo, desde muy joven. Fue al colegio pero no concluyó la enseñanza básica. Una vez iba en un auto y el señor, que allí estaba, le empezó a relatar la historia de su abuelo, decirle que pareciera que ellos eran los dueños de las tierras en Quidico, que quizás existían títulos de merced. Esto la llevó a reunirse con sus hermanos y comenzar a investigar qué era lo que había sucedido con sus abuelos, su familia y sus tierras. Es por ello que, en el 2001, se constituye la comunidad Lorenzo Quintrileo, en donde es dirigenta. También forma parte de la agrupación de mujeres Relmu Witral, la que se constituye como base para que quienes la integran puedan comercializar y ofrecer los productos que ellas mismas hacen. Dentro de la comunidad, tienen un pequeño huerto orgánico familiar, junto con las mujeres que allí habitan. Todo esto, ha hecho que se reconstruya su experiencia como mujer, lo que ha permitido que se mantenga activa.

Luchar por las tierras que pertenecieron a su abuelo ha sido algo importante dentro de su vida y se ha visto involucrada en enfrentamientos directos con Carabineros por la toma de predios que les pertenecían. Esta lucha duró alrededor de 15 años, disminuyendo su intensidad a partir de la recuperación efectiva y legal de algunos de estos predios. 
Tiene cuatro hijos, una mujer -hija de su actual compañero- y tres hombres; además de cuatro nietos. Así también, tiene seis hermanos, y su madre aún está viva. En conjunto con todos ellos han conformado el núcleo central de la comunidad Lorenzo Quintrileo. Tres de sus cuatro hijos forman parte activa de la comunidad. Dos de sus hijos son weichafes o luchadores activos de la causa mapuche. Los recuerdos que tiene de sus padres, es que ambos eran muy estrictos, duros, lo que hizo que casi todos se fueran muy jóvenes de su casa.

De pequeña ha trabajado en el campo, en las tierras de sus papás. Trabajaban cuidando animales con su papá, vendían leña, trigo y papas, entre otros productos. También trabajaban talando "metros ruma" de madera. Esta es una labor que continúa haciendo en el contexto rural en que vive actualmente. Aprendió a tejer desde que era pequeña, lo que le ha permitido desempeñarse informalmente en aquello, hasta hoy. Así también, en conjunto con su madre, siembra y recolecta hierbas (lawen), las que vende para usos medicinales.

Su mamá no es mapuche, su papá sí. Su madre siempre les negó todo vínculo o relación con su cultura, negándoles que se juntaran con "los indios", que hablaran o que realizaran actividades mapuche. Esto generó una aparente pérdida de su identidad. Es por ello que hoy, en conjunto con sus hijos y su esposo, se han encargado de ir reconstruyendo su raíz cultural. Por ejemplo, cuando uno de sus hijos enfermó gravemente, lo llevaron a un médico occidental, y sin presentar mejoría decidieron llevarlo donde una machi a Cañete. Esta autoridad tradicional lo trató satisfactoriamente a partir de hierbas, curaciones y rituales. Sin embargo, debido a su alto costo, decidieron por sus propios medios aprender a trabajar con la medicina de su pueblo. En la actualidad, se tratan sus dolencias con la medicina mapuche. Por otra parte, su casa se caracteriza por respetar ciertas tradiciones mapuche. No tiene calefón, ni baño tradicional; en contraposición a aquello, se calientan con una cocina a leña, la que se encuentra ubicada en el centro de una habitación -que es su cocina y comedor- que es independiente de toda la casa, mientras que su baño es una letrina localizada fuera de la casa. Su hogar está ubicado en relación al recorrido del sol. 


\section{Historia de resistencia 3: Familia Painen, Comunidad Milla Rayén}

Los habitantes de mayor edad de la comunidad recuerdan que llegaron al sector, donde actualmente están asentados, hace unos sesenta años, aproximadamente. Sobre las primeras impresiones que tuvieron del paisaje de la zona, hacen hincapié en que existía una abundante cantidad de vegetación nativa, reflejada en una alta densidad de murtilla, quila, maqui y boldo, entre otras especies. Además, durante esa época comenzaron a producirse los primeros trabajos en agricultura.

Hasta inicios de la década del 70 del siglo pasado, los recuerdos más claros de la comunidad son el de las continuas siembras y cosechas, producidas en un contexto donde no se utilizaban abonos ni fertilizantes, y la infraestructura asociada a los cultivos era más básica que la actual. Por ejemplo, los cercos eran solamente de madera y aún no existían tantas herramientas de trabajo que facilitaran las labores en la tierra.

Con la llegada de los militares al poder y posterior dictadura, en la zona comienzan a presentarse los primeros conflictos notorios y que han perdurado por más tiempo. En primer lugar, una faja de tierra de la familia fue expropiada arbitrariamente para la construcción de un aeródromo que conecta el continente con Isla Mocha. Por otra parte, comienzan a aparecer algunas empresas forestales con las primeras plantaciones de bosque exótico (eucaliptos y pinos).

Durante el periodo comprendido entre los años 1975 y 1985, aproximadamente, las plantaciones de pinos y eucaliptos comienzan a aumentar considerablemente, debido a que las empresas forestales ofrecen incentivos - dineros y/o bonos- a particulares para que planten estas especies en sus terrenos. La posibilidad de generar ingresos monetarios más rápido y la no concientización del posterior daño a la tierra y las especies nativas que generan estas plantaciones, transformaron dramáticamente el paisaje histórico del lugar. 
En la década del 90', en el sector habitado por la comunidad se mantiene la tendencia de las plantaciones de eucaliptus y pinos, pero se va tomando conciencia del daño que generan estas especies en la tierra. Las personas de la comunidad indican que la CONAF era una de las principales instituciones que fomentaba la plantación de estos árboles, incluso más que las empresas forestales. Desde el año 1995, aproximadamente, en la comunidad se comienzan a evidenciar los primeros problemas notorios de escasez hídrica relacionados a las plantaciones de bosque exótico. Se comenzaron a secar los cultivos, obligándoles a usar abonos y a buscar agua en pozos nuevos, no tan cercanos, que mermaron el descenso de los más viejos, entre otras consecuencias. Esta tendencia se mantuvo durante los primeros 10 años del nuevo milenio, viéndose nuevamente alterada luego del terremoto del 2010.

El terremoto ocurrido el 27 de febrero de 2010 generó varias consecuencias negativas en la comunidad. En primer lugar, la casa principal de la familia sufrió graves daños estructurales, obligando a la familia a vivir cerca de tres meses en carpas mientras se levantaba una nueva casa a unos metros de la anterior, totalmente destruida. Asimismo, otras casas también sufrieron daños menos graves pero también requirieron reparaciones. Por otra parte, el fuerte movimiento telúrico ocasionó que varios pozos se secaran producto del gran movimiento de tierras que hubo. Esto generó que la escasez hídrica se intensificara, afectando desde ese año las casas de la comunidad. Esto implicó tener problemas durante largas horas del día para obtener agua, teniendo que priorizarla para las labores más esenciales, como consumir, ducharse, cocinar, utilización del baño, etc.

Debido a toda la problemática que relató la propia comunidad, es que comienza a generarse una concientización en las mujeres sobre su entorno, reivindicando su cultura, por ejemplo, desde la soberanía alimentaria. Así lo expresan algunas de las comuneras:

"Nos empezamos a juntar por la inquietud, queríamos ingresos para nosotras, somos dueña de casa, la idea era hacer un grupo para trabajar. Primero nos juntamos nosotras aquí, luego fuimos con Susana, porque íbamos a comité y nos decían 'es 
que ustedes no pueden entrar porque ya tenemos muchas socias'. Después íbamos a otro grupo y nos decían lo mismo. Entonces ¿por qué tenemos que andar tocando puerta, si podemos hacer algo entre nosotros? Buscamos nombre... a la señora de la municipalidad que nos viniera a constituir, se realizó y hasta ahora todo bien, con alto y bajo pero todo bien".

"De a poco se ha ido tomando conciencia a cuidar el agua. La propia gente plantaba el euca o el pino, porque había financiamiento, recibían. Como ahora estamos con el nativo, los que plantan nativo tienen un financiamiento. Entonces la gente antes lo plantaban tenían su ingreso entonces era plantar y plantar pero no vieron las consecuencias".

"Mi abuelita me enseñó qué plantas usar cuando tuviéramos algún problema medicinal, un problema al estómago, resfrío o, a veces, sacamos alguna plantita para el mate".

"Yo ahora a mi chiquitita la llevo al bosque, le digo que hay que respetar la tierra, que no hay que cortar algunos árboles, que el euca es malo y se puede quedar sin agua”.

\section{Historia de resistencia 4: Carmen Carrillo, Comunidad Alto Primer Agua}

Carmen es una mujer dirigente social y preside la junta de mujeres de Tirúa, ha pertenecido al Alto Primer Agua desde que nació. Es participe de PROEMPLEO y jefa del invernadero construido en su propiedad. Como guía del invernadero, conoce todo lo que se refiere a la administración, construcción y ejecución del huerto. La estructura del invernadero en base a botellas recicladas se construyó con la participación de cuatro personas, en un tiempo estimado de cuatro meses. Para ello, nos cuenta Carmen, "se utilizaron 3.800 botellas plásticas, que son parte de la estructura base del invernadero. Su funcionamiento fue a partir de mayo del 2015. Este invernadero busca la alimentación saludable, por medio de semillas orgánicas y un compost de residuos orgánicos para plantar". Como dirigente social, es una mujer comprometida con las prácticas mapuche, incluso cuando no 
pertenece originariamente al pueblo. Se ha transformado en la organizadora de dos trafkintus -ceremonia de intercambio de semillasen conjunto con mujeres mapuche del sector. Ello demuestra la integración de prácticas culturales y la importancia de mantenerlas en territorios degradados, en este caso, conservar las semillas nativas para luego ser plantadas en el área de Alto Primer Agua. Carmen explica el vínculo y la articulación con la cultura y prácticas mapuche en el trabajo con las semillas nativas:

"Hemos replicado la ceremonia con ayuda de machis de la zona, generando lazos con otras comunidades y siendo invitadas a ceremonias fuera de la ciudad. Por medio de estas es que se recolectan nuevas semillas orgánicas para la producción del invernadero, además de realizarse recolección en nuestros sectores aledaños".

Consciente de las problemáticas del lugar, Carmen reconoce su preocupación por el abastecimiento de agua en el sector, más aún con la instalación del vivero que necesitará mayor cantidad de agua: "el abastecimiento de agua en verano es escaso y muy reducido, y puede verse perjudicado el proyecto por esta razón. Nuestros pozos no son lo suficientemente grandes para recolectar lo necesario y abastecernos, tanto en infraestructura como en falta de agua en el río". De esta manera, también hace una autocrítica sobre la instalación de monocultivo forestal, puesto que "muchos cometimos el error de aceptar el incentivo de plantación forestal y tenemos grandes áreas de Eucalipto". De esta manera, reconoce que el daño al suelo, a los ríos y esteros es perjudicial para el abastecimiento de agua del sector. Carmen ha logrado ser una persona capaz de concientizar desde su rol como dirigente sobre la importancia de proteger las nacientes de aguas. Y así lo expresa con claridad: "el vivero también quiere responder a la recuperación de aguas, plantando en las nacientes de los ríos y protegerlos". 


\section{Ideas al cierre}

El avance de la frontera forestal de monocultivo amenaza seriamente los recursos naturales de Tirúa y a las especies nativas. Esto se debe a que las actividades forestales no son sostenibles en el territorio, dado el predominio de especies introducidas como el pino y el eucalipto, que abarcan algo más del $45 \%$ de la superficie comunal. Si bien los comuneros, en general, tienen pocas nociones económicas de las actividades que podrían hacerse para aprovechar los productos del bosque nativo, en estos últimos años ha existido una mayor concientización sobre la importancia de las especies nativas, en tanto fuentes de reforestación y restauración de los suelos degradado y que, en definitiva, pueden significar una alternativa al monocultivo. Ejemplo de esto es la construcción de viveros como fuente de producción, en un futuro próximo, de especias nativas.

También, mujeres del territorio señalan la necesidad de rescatar plantas medicinales, murtilla y maqui con fines artesanales y gastronómicos. Si bien la superficie de bosque nativo alcanza solo un $12 \%$ de la superficie comunal, relegados principalmente a fondos de quebradas, esteros y nacientes de ríos, en el último tiempo han comenzado iniciativas que buscan cercar los fondos de quebrada. El objetivo es proteger el renoval de bosque nativo allí presente y resguardar un ecosistema que mejora la retención del agua y da cobijo a una mayor diversidad de fauna nativa.

En los predios actuales, el agua es extraída principalmente de pozos que se encuentran muy cercanos a las nacientes de los esteros. Estas aguas no poseen algún tipo de tratamiento, debido a que la extracción se realiza de manera directa. Entre los diversos usos, se contempla el consumo humano y tareas hogareñas como lavar ropa, bañarse y para dar de beber al ganado y gallinas domésticas. En época de verano, algunos pozos se secan, puesto que muchos de los esteros comienzan a correr bajo tierra y las familias deben recurrir a racionalizar la cantidad de agua, además de usar pozos alternativos que tienen una menor calidad. 
Algunas familias ven como una alternativa real de ingreso cortar y vender "palos", comercializar productos nativos en bruto y manufacturados como especies arbóreas, florales y arbustivas nativas, artesanía en ñocha y lana, además de productos elaborados como mermeladas y chicha de manzana. Una de las ventajas que desean explotar es poder utilizar la localización estratégica del aeródromo de Tirúa que realiza viajes a la Isla Mocha durante todo el año, para poder captar el flujo de turistas. Aquí, los invernaderos donde se reproducen árboles nativos, a través de almácigos, patillas y esquejes, adquieren un valor especial. El desafío pendiente para este tipo de resistencia es mejorar los canales de comercialización y los medios de difusión.

Entre los beneficios a nivel territorial, se encuentran poder diversificar la oferta del comercio de artesanías y productos nativos, generando, a su vez, desarrollo local por medio del mercadeo de productos con identidad cultural de la comuna y comercio justo. Esto permitiría heredar el conocimiento y técnicas a las nuevas generaciones y fortalecer la identidad cultural de la población lafkenche.

De esta forma, se han producidos hitos en la Red de Mujeres del Lafkenmapu, que dan cuenta de eventos, iniciativas y logros, como los "Intercambios de Semillas" el 20 de abril y el 22 de octubre en Mercado Lafkenmapu, Tirúa. La participación en otros territorios en trafkintu -tradición del Pueblo Mapuche donde las personas intercambian productos y comparten en una ceremonia- el 10 de abril en Galvarino, el 11 de mayo en Temuco y el 10 de octubre en Imperial. Finalmente, ocho mujeres participan activamente del puesto en el Mercado Lafkenmapu, bajo el nombre de Kelluwün Domo, comercializando los productos que producen o elaboran, valorando saberes propios y cuidando el medio ambiente. 


\section{Bibliografía}

Agosto, P. y Briones, C. (2007). Luchas y resistencias mapuche por los bienes de la naturaleza. En OSAL (Buenos Aires: CLACSO) Año VIII, No 22, septiembre.

Donoso, P., Romero, J., Reyes, R. y Mujica, R. (2015). Precedentes y efectos del neoliberalismo en el sector forestal chileno y transición hacia un nuevo modelo. En Pinol (Ed.) Democracia versus neoliberalismo. 25 años de neoliberalismo en Chile. LOM Ediciones.

FUCOA. Fundación de Comunicaciones, Capacitación y Cultura del Agro, . (2014). Mapuche. Serie introducción histórica y relatos de los pueblos originarios de Chile. Santiago.

Gonzalez, M. (2015). ¿Agua para quién? Escasez hidrica y plantaciones forestales en la provincia de Arauco. Chile. Recuperado de http://www.mapuexpress.org

INE (2007). Censo agropecuario y forestal. Resultados por comuna. Chile. Recuperado de http:// http://historico.ine.cl

Martínez, V. (2006). El enfoque comunitario. El desafío de incorporar a la comunidad en las intervenciones sociales. Santiago: Universidad de Chile.

Hasen, F. N. (2012). La noción de Kume mogñen en el Pueblo Mapuche: aproximaciones desde un enfoque ecosistemico . Kula. Antropólogos del Atlántico sur, 96-114.

Hasen, F. N. (2014). Desarrollo y BuenVivir desde un Enfoque Ecosistemico: La experiencia local de Lago Neltume, Chile. Sustentabilidades, 10, 1-23.

Pineda, E. (2012). Dominación y emancipación en el Pueblo Mapuche. El pensamiento de la Coordinadora Arauco Malleco. SubHistoria $N^{\circ} 4,43-71$.

PLADECO. I. Municipalidad de Tirúa. (2009). Plan de desarrollo comunal. Tirúa. Región del Bio Bío

Programa Chile Sustentable. (2004). Impactos ambientales en Chile: Desafios para la Sustentabilidad. LOM Ediciones.

Rubilar, G. y Roldán, A. (2014). Áreas de Desarrollo Indígena: estudio de caso del ADI Puel Nahuelbuta, como estrategia de las políticas públicas en el mundo mapuche. Universum, vol.29, n.2, pp.253-276. 